\title{
Anton R. v. Noga Mars $\dagger$
}

Wieein Soldatim Schützengraben ist Professor Mars am 23.April 1918 versehieden. Eine kaum sichtbare Wunde am Finger, durch die Bürste beim Waschen der Hände verursacht und bei derTJntersuchung einer Pa-tientin infiziert, bildete die Eingangspforte zur bösartigen Phlegmone. In Wenigen Tagen erlag Mars der Krankheit, - - gerade er, Weicher das ganze Leben das Banner der Anti- und Aseptik so hoch getragen hat und Weleher den Kampf mit Mikroorganismen zu seinem ersten und Wichtigsten Lebens-bestreben machte.

Anton v. Mars, in Chrz\%stów in Polen im Jahre 1S51 geboren, absol-vierte die Mittel-und Hochschule in Krakau und erlangte hier 1875 das Doktordiplom. Nach speziellen Studien in der Geburtshülfe und Gynä-kologiebei Braun inWienund Czy'zewícz in Lemberg vtxivã e or im Jahre 1878 bei Madurowicz Assistent der Universitäts-Frauenklinik in Krakau. Er habilitierte sich 1880 mit der Arbeit über die Durchgangsíähigkeit der Placenta für organisierte Stoffe. Um seines Lehramtes selbständig walten zu können, gründete der junge Dozent auf eigene Kosten ein Laboratormm für bakteriologische, chemische und mikroskopische Untersuchungen. Dort hielt er seine Vor-lesxingen und leitete Übungen in geburtshülflichen Operationen. Als aiißerordentlicher Professor in Krakau publizierte er im Jahre 1890 die Ergebnisse eines Gefrierdurchschnittes einer kreißenden Fralı auf G $\pi$ ind einer mühevoll $\theta$ n, dama $1 / 8$ noch Wenig b $\theta$ kannten Untersuchungsmethode.

Seinem Lehramte roit ganzer Seele ergebon, widmete sich nun Mars mit größtem Eifer seiner Kanzel und fesselte seine Hörer durch seine hervorragende Vortragskunst. Ein Meister zugleich im Zeichnen und Moðellieren, bot er den Schülern nicht nur das klare Wort, sondern auch Bilder und Modelle, Welche in hohem Maße das Lernen erleichtern. Er faßte sie zusammen zu seinem „Atlas der praktischen gynäkologischen Operationen”, der ihm allgemeinen Beifall und Dank sowohl auf dem Kongresse der Gynäkologen in Wien, Wie im internationalen Kongresse in Rom eintrug.

Im Jahre 1895 erschien seine Arbeit über Entwicklung der Placenta.

Zum ordentlichen Professor ernannt, übernahm Mars die Leitung áo $\tau$ Hebammenschule in Krakau und der gynäkologischen Abteilung des Krankenhauses. Jetzt hatte er seine ersehnte eigene Arbeitsstätte. Als Ergebnisse seiner Tätigkeit erschienen zahlreiche Veröffentlichungen all $1 \frac{8}{8}$ strong Wissenschaftlich, alle mit eigenartiger Gründlichkeit verfaßt, alle auf unumstößliche Experimente oder Bcoachtungen gestützt. Über 90 im ganzen befassen sie sich mit alien Teilen der Fachwissenschaft.

Anton R. v. Noga Mars †.

Das Jahr 1898 stellte Mars an die Spitze der neu errichtet $\beta \propto$ Prauen-klinik in Lemberg. Er bekam ßine neue Klinik und brachte in kurze $\Gamma$ Zeit rege Tätigkeit und warm pulsierendes Leben hinein. Sein únermüdlicher Fleiß, seine hohe Wissenschaftlichkeit, sein Takt und sein zielbeWußtes Auítreten führten bald die Klinik zu raschen Fortschritten. ,,Virtutis laus omnis in actione consistit" war seine Devise \md treu derselben kannte er keine Ruhe. Der Erfolg entsprach dieser unermüdlichen Arbeit. Mars gab seinem Vaterland eine große Anzahl tüchtig ausgebildeter 
Geburtshelfer und Frauetuäzte. Ein großer Kreis genesener Frauen verbreitete den Ruf der Lemberger Klinik Weithin. -

Als eine bedeutungsvolle Anerkennung seines Wirkens erhielt er von

D. v. Ott-St. Petersburg den Auftrag, fürden internationalen Gynäkologen-

Kongreß 1910 ein polnisches Komitee zu organisieren. Auch auf dem 1912

in Berlin tagenden Kongreß erstattete Mars für das polnische Komitee ein

erschöpfendes Referat über die chirurgische Behandlung der Peritonitis.

Damit trat die polnische Gynäkologie zum ersten Male mit einem selb-

ständigeti Komitee in die Weltarena. S

Als im Jahre 1911 die Gesellschaft der polnischen Gynäkologen ge-gründet Wurde, Wurde Mars

einstimmig zum Präsidenten erWählt und leitete sie bis an sein Lebensende.

Im Jahre 1901 bekleidete er das Amt des Dekans, - - im Jahre 1908 war er Rektor der Lemberger Universität.

Trotz der zahlreichen Fachbeschäftigungen, trotz der großen Praxi« und trotz der vnermüdlichen wissenschaftlichen Tätigkeit fand Mars doch noch Zeit, auch im allg $\beta$ meinen Interesse zu wirken. Als Sanitätsrat und Landtagsabgeordneter war er Sanitäts-R $\theta$ f erent zahlreicher wichtigcr Unter-nehmungen tmd übte einen großen Einfluß atif das Sanitätswesen Galiziens aus.

Es kam die große Kriegszeit. Lembírg geriet in russische Hande, die gynäkologische Klinik verWandelte sich in ein Reservespital des Roten Krouzes und Mars mußte mit schWerem $\mathrm{H} \beta$ Гzzen seine Arbeitsstätte auf einigo Zeit verlassen. Er ging na $<1 / 8$ Wien, entfaltete auch dort sofort eine rastlose Tätigkeit, indem er ein gyn äkologisches Ambulatorhim für Flücht-linge gründete. Kaum einige Tage nach Lembergs Befreiung war er schon an Ort und Stelle, um seine Vorlesiingen wieder aufnehmen zu können, ohne Assistenten, Welche alle im Kriegsdienst standen, ohne Klinik, Welehe KriegszW $\theta$ cken diente, ohne Material und Instrumßnte. Nur auf seinZimmer und das Ambulatorium beschränkt, führte erdoch die Arbeit, um die Hörer miter dem Mangel der Klinik nicht leiden zu lassen. Als es ihm endlich ge-lang, den UniversitätsUnterricht wieder auf normalen Weg zu bringen, erreichte ihn plö†·zlich das tückische Ende. Seine sterbliohen Reste Wurden auf sein Erbgut Limanowa überführt, und eine unzählige Schar seiner Freunde und Verehrer gab ihm das letzte Geleíte.

Im Sehatten stiner geliebten Bätime fand er Ruhe, die er im Leben niemals genossen. Sein Name und sein Andenken bltiben ewig in den Herzen aller, die ihn je gekannt, denn stets war er nicht nur ein unerreichbarer Meister und Lehrer, aber auch ein treutr, wahrerFreund, der keine Mühe schelıte, um Gutes zu tun! - Adam Gzyzewicz. 